\title{
Adult Spinal Deformity: Current Concepts and Decision-Making Strategies for Management
}

\author{
Hong Jin Kim ${ }^{1,}$, Jae Hyuk Yang ${ }^{2,}$, , Dong-Gune Chang ${ }^{1}$, Se-Il Suk ${ }^{1}$, \\ Seung Woo Suh', Kwang-Sup Song ${ }^{3}$, Jong-Beom Park ${ }^{4}$, Woojin Cho ${ }^{5}$ \\ ${ }^{1}$ Department of Orthopaedic Surgery, Inje University Sanggye Paik Hospital, Inje University College of Medicine, Seoul, Korea \\ ${ }^{2}$ Department of Orthopaedic Surgery, Korea University Guro Hospital, Korea University College of Medicine, Seoul, Korea \\ ${ }^{3}$ Department of Orthopaedic Surgery, Chung-Ang University Hospital, Chung-Ang University College of Medicine, Seoul, Korea \\ ${ }^{4}$ Department of Orthopaedic Surgery, College of Medicine, The Catholic University of Korea, Seoul, Korea \\ ${ }^{5}$ Department of Orthopaedic Surgery, Montefiore Medical Center, Albert Einstein College of Medicine, Bronx, NY, USA
}

Adult spinal deformity (ASD) is characterized by three-dimensional abnormalities of the thoracic or thoracolumbar spine that exerts significant impacts on the health-related quality of life (HROOL). With the important effects that deformity of the sagittal plane exerts on the HROoL, there have been paradigm shifts in ASD evaluation and management. Loss of lumbar lordosis is recognized as a key driver of ASD followed by reducing kyphosis, pelvic retroversion, and knee flexion. The Scoliosis Research Society (SRS)Schwab classification reflects the sagittal spinopelvic parameters that correlate pain and disability in ASD patients. Although the SRS-Schwab classification provides a realignment target framework for surgeons, a structured patient-specific systemic approach is crucial for the process of decision-making. ASD management should be focused on restoring age-specific harmonious alignment and should consider the comorbidities and risk factors of each patient to prevent catastrophic complications and enhance the HROoL.

Keywords: Adult spinal deformity; Sagittal imbalance; Health-related quality of life; Surgical treatment; Complications

\section{Introduction}

Adult spinal deformity (ASD) is a heterogeneous spectrum of abnormalities of the lumbar spine or the thoracolumbar spine that occurs in adult patients. Specific ASD diagnoses include primary degenerative sagittal imbalance, iatrogenic spinal deformity, and adult spinal scoliosis $[1,2]$. With improved medical care, increasing life expectancy, and a higher proportion of healthy elderly subjects, the incidence and prevalence of ASD is on the rise [2-4]. Schwab et al. [3] reported a high prevalence of
$68 \%$ for ASD in adults aged $>60$ years.

ASD is characterized by a series of progressive, asymmetric, and degenerative changes that may cause neural compression. This degeneration leads to pathological changes and load-bearing abnormalities via the asymmetric collapse of the motion segments [1-4]. The condition may further lead to back pain or cause neurologic symptoms and progressive deformity, each of which causes an imbalance of the structural support of the spinal column. Specific ASDs include scoliosis, sagittal malalignment, kyphosis, spondylolisthesis, rotatory subluxation, and axial

Received Nov 9, 2020; Revised Nov 10, 2020; Accepted Nov 11, 2020

Corresponding author: Dong-Gune Chang

Department of Orthopaedic Surgery, Inje University Sanggye Paik Hospital, Inje University College of Medicine, 1342 Dongil-ro, Nowon-gu, Seoul 01757, Korea

Tel: +82-2-950-1284, Fax: +82-2-950-1287, E-mail: dgchangmd@gmail.com

*These authors equally contributed to this study as the co-first author. 
plane deformity [2,5].

In the view of treatment considerations, it is crucial to consider regional and global patterns of scoliosis as well as sagittal and coronal imbalances. Several spinopelvic parameters correlate with ASD clinical outcomes that suggest that these can guide the process of decision-making with respect to the choice of surgery and non-operative treatment [1-6].

Previously, most surgeons focused on conservative ASD management options owing to increased perioperative morbidity and a higher incidence of neurological deficits associated with operative management [2]. However, surgical intervention has become the mainstay of ASD treatment, resulting from the consideration of the importance of spinopelvic alignment, advances in surgical techniques, and owing to the fact that medical treatment does not significantly improve the health status of ASD patients [4]. This manuscript discusses the current concepts of ASD, starting from pathophysiology to decision-making strategies for ASD treatment.

\section{Definition and Pathophysiology of Adult Spinal Deformity}

Spinal deformity can be defined as the spinal curvature or alignment that deviates from the normal limits [2]. ASD can include any combination of spinal deformities affecting the axial, coronal, and sagittal planes. In the coronal plane, scoliosis is defined as a lateral spinal curvature $>10^{\circ}$ with a resulting concomitant rotational deformity in the axial plane and kyphosis or loss of lordosis in the sagittal plane. With an increase in the average age of the general population and a rise in the life expectancy of the elderly, the ASD prevalence is expected to escalate further, with a concomitant increase in the associated social and economic burden $[4,5]$.

ASD was believed to result from accumulated degenerative changes that occur with age. Degenerative bone and soft-tissue changes cause radiculopathy or instability via spondylolisthesis or rotatory subluxation that induce spinal stenosis [1]. The initial degenerative process is a loss of absorbable function of the intervertebral discs that is characterized by reduced disc height, loss of water and proteoglycan content, and increased enzyme degradation. Subsequently, the pathological changes of vertebral and facet joints increase the load on the anterior part of the vertebral joints and cause arthritic changes to posterior elements, which induce bone remodeling and instability $[1,7,8]$.

ASD with sagittal imbalance can be summarized as the spine losing the sagittal curvature that manifests as loss of lordosis, forward leaning of the trunk, and posterior rotation of the pelvis. Diminution of the lumbar lordosis (LL) can be caused by several factors, including degenerative changes, scoliosis, and iatrogenic changes. LL loss tilts the body forward, resulting in an increase in the pelvic incidence (PI)/LL mismatch and the sagittal vertical axis (SVA) [7-9]. In order to maintain an erect posture, patients with sagittal imbalance use the following series of compensatory mechanisms: the head and neck tilt back to level the gaze, and the thoracic spine straightens to reduce kyphosis, with concurrent pelvic retroversion and knee flexion $[7,10]$. For aging subjects with ASD, surgery focuses on correcting sagittal imbalance more than scoliosis because sagittal imbalance is associated with more severe pain and disability. Coronal imbalance can also lead to back pain and impaired function; however, it is more commonly associated with an undesirable appearance [11]. However, the ideal treatment of ASD must consider both, the sagittal and coronal parameters during surgical treatment.

\section{Radiological Assessment of Adult Spinal Deformity}

The alignment of the spine is important for the maintenance of an upright posture, protection of neural elements, and stability of the axial skeleton [1,2]. In recent times, radiological assessment has been critical in the analysis of pain and disability and surgical planning. Therefore, it is crucial to capture radiographic images (with the patient in the correct posture) that can confirm imaging landmarks. Usually, imaging is performed using the Cobb method.

In order to capture a proper whole-spine lateral view, a $30-90-\mathrm{cm}$ vertical film should be used and should be uniformly $182.88 \mathrm{~cm}$ away from the subject. The knee and hip joints should assume natural postures (full extension is not required). Although there remains a controversy about arm positioning, the following description should be followed to prevent external factors from masking spinal deformities and allow the visualization of critical radiological landmarks; the patient should be free-standing without support with the hands in the clavicle position (i.e., elbows fully flexed, hands in a relaxed fist posture, 
wrists flexed, and the proximal interphalangeal joints resting into the supraclavicular fossae) $[1,6]$.

In radiographs captured in the sagittal and coronal planes, regional, global, and sagittal spinopelvic parameters are used to assess sagittal alignment.

\section{Regional parameters}

There are various opinions for the measurement sites for regional parameters. Certain disagreements exit; however, the following definitions are usually applied with consideration of the anatomical shape of the spine. Regional parameters include cervical lordosis (CL; Cobb's angle between the inferior endplate of the $\mathrm{C} 2$ and the inferior endplate of C7), thoracic kyphosis (TK; Cobb's angle between the superior endplate of T4 and the inferior endplate of T12), thoracolumbar kyphosis (Cobb's angle between the superior endplate of T10 and the inferior endplate of L2), and LL (Cobb's angle between the superior endplate of L1 and superior endplate of S1). Berthonnaud et al. [12] reported a high correlation of CL with LL in terms of pelvic parameters (i.e., pelvic tilt [PT], sacral slope [SS], and PI), with a low correlation with TK. Furthermore, they describe the existence of a chain interaction between pelvic parameters and LL, then LL and TK, and then TK and CL (Fig. 1) [12]. As per this observation, they also suggested that changes in the shape or orientation at a functional level will exert a direct influence on the adjacent segment. Knowledge of these normal relationships is vital for the comprehension of sagittal balance in normal and pathologic conditions of the spine and pelvis.

\section{Global parameters}

Global parameters include the SVA (the distance between a plumb line from the center of the C7 vertebra to the posterior superior corner of the sacrum) and the T1 pelvic angle (angle formed by the intersection of a line extending from the center of the T1 vertebra to the center of the bicoxofemoral axis that is the center point of the overlap between the femoral heads, and a line that extends from the center of the bicoxofemoral axis to the middle of the S1 superior endplate) (Fig. 1).

\section{Sagittal spinopelvic parameters}

Sagittal spinopelvic parameters include the PI (the angle between a line perpendicular to the sacral endplate and a line connecting the center of the femoral head and the center of the sacrum), PT (the angle between a vertical reference line and the line joining the midpoint of the sacral plate to the center of the femoral heads), and SS (the angle between a horizontal reference line and the upper sacral endplate) (Fig. 1) [10-15].

PI is a morphological variable; thus, it is minimally affected by age- and position-related changes. The parameters of PI describe the pelvis orientation and contributes to the LL. Sagittal malalignment must be harmonized by LL and PI. Age-related LL loss induces a PI/LL mismatch that is a crucial prognostic factor in terms of outcomes and disability. Therefore, the fundamental goals of deformity surgery are the restoration of the lordotic curve and PI/LL match. However, PT and SS are dynamic variables. A low $\mathrm{PT}$ reflects on the pelvis rotated anteriorly (anteversion), while a high PT reflects on the pelvis rotated posteriorly (retroversion). SS determines the position of the lumbar spine. A low SS reflects on a horizontal sacrum, while

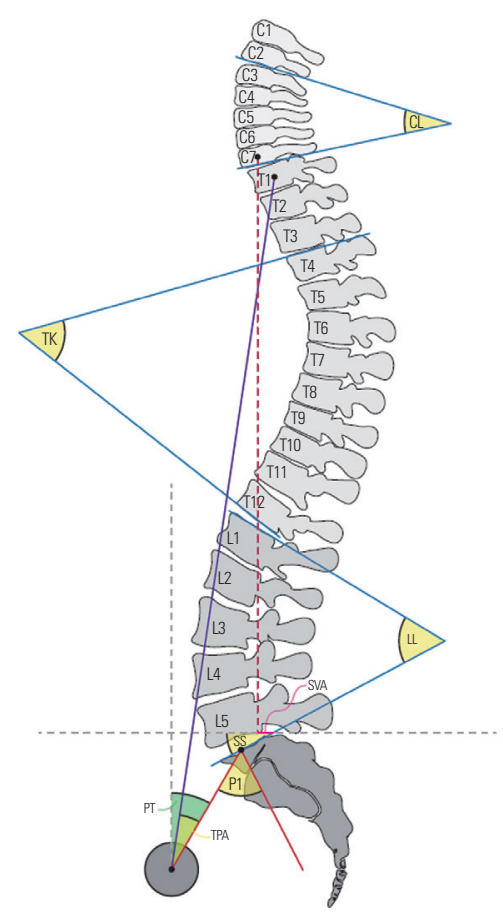

Fig. 1. The important radiological determinants of adult spinal deformity. The important radiological determinants include regional variables (CL, TK, and LL), global variables (SVA and TPA), and sagittal spinopelvic variables (PI, PT, and SS). The degree of disruption of the normal spinopelvic morphology is determined via the calculation of spinopelvic mismatch, which is the difference between PI and LL. CL, cervical lordosis; TK, thoracic kyphosis; LL, lumbar lordosis; SVA, sagittal vertical axis; TPA, T1 pelvic angle; PI, pelvic incidence; PT, pelvic tilt; SS, sacral slope. 
a high SS reflects on a vertical sacrum. Mathematically, the following equation has been established: $\mathrm{PI}=\mathrm{PT}+\mathrm{SS}$. Therefore, pelvic retroversion (high PT) will be associated with a vertical sacrum (low SS) [10].

Sagittal spinal alignment parameters, including SVA, $\mathrm{TK}$, and LL, are routinely used in the assessment and management of ASD. Moreover, spinopelvic alignment is an important determinant of the regional and global sagittal alignment of the spine. Schwab et al. [3] reported that a PT $>22^{\circ}$ and a PI/LL mismatch $>11^{\circ}$ are thresholds for spinopelvic regional alignment associated with increased pain and disability.

\section{Coronal parameters}

In terms of the coronal plane, Cobb's angle and axial rotation of vertebrae in the apical region can be measured for the assessment of scoliotic curves and type as regional parameters. The global parameters for the assessment of coronal alignment are the distance between the $\mathrm{C} 7$ plumb line (C7PL; a vertical line from the center of the C7 vertebral body) and the central sacral vertical line (CSVL; a vertical line from the center of the sacrum) $[7,8]$.

\section{Classification of Adult Spinal Deformity}

Classification systems for ASD demonstrate that clinical outcomes are vital for the accurate characterization of specific disorders that would facilitate evidence-based decision-making and management [16,17]. In 2005, Aebi [18] proposed a classification system based on etiology that is uniquely helpful in understanding the natural history of ASD. However, Bess et al. [16] proposed a new classification system that was based on "high-impact, clinically significant radiographic parameters" through a prospective study that investigated the clinical outcomes $[17,19,20]$. In 2012, the Scoliosis Research Society (SRS) Adult Deformity Committee revised the previously proposed system in order to include the pelvic parameters owing to substantial correlations with health-related quality of life (HRQoL) $[17,19]$. Currently, the SRS-Schwab adult spine deformity classification is considered the standard system because all of its modifiers include spinopelvic parameters and owing to a strong correlation with the HRQoL scores $[1,16]$. The SRS-Schwab system involves a coronal curve type and sagittal modifiers, including PI minus LL, SVA, and PT.
Coronal curve type is based on the location and the Cobb's angle of the scoliotic curves. These criteria apply to curves $>30^{\circ}$. The modifiers include type $\mathrm{T}$ (thoracic only; thoracic major curve $>30^{\circ}$ at the apical level of $\mathrm{T} 9$ or higher), type L (TL/lumbar only; isolated thoracolumbar or lumbar curve $>30^{\circ}$ at the apical level of T10 or lower), type $\mathrm{D}$ (double major curve with thoracic and thoracolumbar/lumbar curve $>30^{\circ}$ ), and type $\mathrm{N}$ (normal; no definite coronal deformity) [16-19].

Sagittal modifiers are PI/LL mismatch, global alignment, and PT. First, PI/LL mismatch is the degree of disharmony between PI and LL. If PI minus LL is $<10^{\circ}$, the modifier is " 0 "; if PI minus LL is between $10^{\circ}$ and $20^{\circ}$, the modifier is " + "; and if PI minus LL is $>20^{\circ}$, the modifier is "++." Increases in PI/LL correlate with decreases in SRS score and 12-item Short Form Health Survey as well as increases in Oswestry Disability Index (ODI) with important implications for patient outcomes and disability. Therefore, these modifiers are important for surgical planning in patients in order to achieve correct postoperative sagittal alignment. Furthermore, global alignment is calculated by using SVA. If SVA is $<40 \mathrm{~mm}$, the modifier is " 0 "; if the SVA is $40-95 \mathrm{~mm}$, the modifier is "+"; if the SVA is $>95 \mathrm{~mm}$, the modifier is "++." Increases in SVA are correlated to pain and disability. Finally, PT, as a positional parameter, reflects compensatory pelvic retroversion to maintain upright balance if the degree is $>20^{\circ}$. If PT is $\left\langle 20^{\circ}\right.$, the modifier is " 0 "; if the degree is $20^{\circ}-30^{\circ}$, the modifier is "+"; if the degree is $>30^{\circ}$, the modifier is "++." Increases in PT correlate to pain and disability [16-20].

As per a multicenter prospective study that investigated the correlation between spinopelvic parameters and HRQoL, Schwab et al. [3] reported the predictive ODI is $\geq 40$ when PI minus LL is $\geq 11^{\circ}$, SVA is $\geq 47 \mathrm{~mm}$, and PT is $\geq 22^{\circ}$. Terran et al. [21] also reported significant differences in the clinical outcomes between sagittal modifiers ( $p<0.001$ for all). The mean ODI values with “++" modifiers compared with " 0 " modifiers were as follows: PT (41.7" versus $\left.24.8^{\circ}\right)$, SVA $(47.8 \mathrm{~mm}$ versus $25.6 \mathrm{~mm}$ ), and PI/ LL mismatch $\left(44.2^{\circ}\right.$ versus $\left.24.5^{\circ}\right)$. Furthermore, Smith et al. [15] found that the ODI improved with changes in PT and SVA; SRS-22 improved with changes in PT, SVA, and PI/LL mismatch; and 36-item Short Form Health Survey improved with changes in SVA and PI/LL mismatch. Even if these clinical values are included, the classification is limited because it does not provide a specific choice of surgical technique or determination of fusion level $[22,23]$. 


\section{Spectrum of Adult Spinal Deformity}

\section{Adult sagittal imbalance}

1) Primary degenerative sagittal imbalance (lumbar degenerative kyphosis)

Lumbar degenerative kyphosis (LDK) is considered as a form of sagittal imbalances caused by lumbar kyphosis or a marked loss of LL [24,25]. First, Takemitsu et al. [26] suggested that LDK that is reportedly common among middle-aged women in Asian countries because of unique lifestyle factors, such as prolonged crouched posture and activities of daily living on the floor. The cause can be degenerative changes, including disc space narrowing, collapsed vertebral bodies due to osteoporosis, and atrophy of the lumbar extensor muscles [27].

Recently, this disease has been described as a subtype of flat back syndrome that is characterized by primary degenerative sagittal imbalances because it is not limited to the lumbar curvatures [28]. Takemitsu et al. [26] classified four types of spinal curvatures. Type 1,2, and 3 are sagittal thoracic compensated groups, and type 4 is the sagittal thoracic decompensated group [24]. The notable symptoms referred to the four following cardinal signs: forward stooping of the trunk, inability to hold things in front of one's self, elbow corns, and difficulty in climbing slopes [29].

Various methods have been attempted in order to evaluate degenerative sagittal imbalance. Shiba et al. [30] performed a three-dimensional (3D) gait analysis test to evaluate the degree of degenerative kyphosis. In this study, they reported that it is possible to identify the shape of the patients' intensifying sagittal imbalances during walking. Bae et al. [31] recommended taking whole-spine lateral images after the subjects walked for 10 minutes to assess sagittal spinal imbalance that may be compensated for static images. Moreover, low-dose radiation systems (EOS system; EOS Imaging, Paris, France) are being used. This system can take both anterior and lateral images and can perform 3D reconstruction of the vertebral body; this eliminates distortion when radiographic images are acquired with pelvic rotation.

Previously, first-line treatment was conservative management, including nonsteroidal anti-inflammatory medications and exercise [32]. Recently, based on a multicenter prospective study, Bridwell et al. [27] reported that common medical treatments did not affect the patient's quality of life. However, operative interventions are associated with significant improvements in the ODI, SRS, and HRQoL measures $(p<0.001)$ [4]. Indications for operative care are not absolute; however, it should be considered in the context of patient-specific factors. Generally, deformity progression, neural compromise, pain, and functional limitations unresponsive to medical interventions are the primary indications for the surgical treatment of ASD $[4,24]$.

\section{2) Iatrogenic spinal deformity}

Iatrogenic spinal deformity is associated with extensive spinal fusion that produces symptomatic loss of lordosis [1]. Flat back syndrome, initially described by Doherty [33], is a postural disorder accompanied by symptomatic fixed forward inclination of the trunk caused by LL loss after posterior spinal fusion for scoliosis [34]. Previously, the most common cause of iatrogenic flat back syndrome was the use of distraction-type instrumentation, including Harrington and Cotrel-Dubousset devices [34,35]. However, with the use of the segmental pedicle screw fixation system, insufficient correction of LL has been the main cause of iatrogenic flat back syndrome [34]. Although these instruments improve the coronal curvature, they reduce TK and LL via distraction of the thoracolumbar spine. Postoperative changes include the compensatory mechanisms that work on adjacent distal unfused levels, such as paraspinal muscle fatigue, pelvic retroversion, hip extension, knee flexion, and hyperextension of the cervical spine [36]. These abnormal postures lead to the development of lower back pain and gait disturbances $[34,35]$. Iatrogenic flat back syndrome can be categorized as per the compensation. Type 1 is segmental deformity that is accompanied by loss of lordosis and maintenance of normal sagittal balance. Type 2 is global deformity that is characterized by loss of lordosis and substantial fixed positive sagittal imbalances [36]. Full-length standing anteroposterior and lateral radiographs of the spine with the hip and knee extended are required for the radiological evaluation of flat back syndrome.

Preoperative assessment and prevention of risk factors for reducing LL are important for preventing iatrogenic flat back syndrome. Before spinal fusion of long level, adequate radiological assessment of preoperative sagittal imbalance and rotational and coronal deformities are needed. In terms of radiological assessment, the most important factor is the correction of sagittal imbalances. 
In intraoperative planning, surgical positioning affects LL maintenance. The patients should be positioned on Jackson or Wilson frames, with the hips fully extended to prevent iatrogenic flat back syndrome because lordosis is decreased by $26 \%$ when the hips are flexed about $33^{\circ}$ [34]. Recently, a segmental pedicle screw fixation system has been widely used because it facilitates improved rotational control of scoliosis and three-column fixation, which properly restores sagittal alignment $[34,35]$.

\section{Adult spinal scoliosis}

Adult spinal scoliosis is a $3 \mathrm{D}$ deformity defined as a coronal deviation $>10^{\circ}$. It is a rotational disorder that induces loss of lordosis with a relatively flexible thoracic compensatory curve $<30^{\circ}$. This scoliotic curve progresses with advancement in age. The risk factors for curvature progression include increased intervertebral disc degeneration, apical lateral vertebral translation $\geq 6 \mathrm{~mm}$, and Nash and Moe's grade II/III apical vertebral rotation [37].

This chronic condition results from degenerative changes, leading to pain, radiculopathy, and instability. Pain is the most common symptom of adult spinal scoliosis. Although it is localized over the convex curvature; further, muscle fatigue due to compensatory coronal and sagittal imbalance can lead to spreading of the pain. Disc rupture or facet hypertrophy narrowing in the lateral recess because of degenerative changes can induce adjacent nerve root compression, resulting in radiculopathy [38]. In terms of deformity and instability, "rip-on-hip" discomfort with positive sagittal balance are the typical signs of adult degenerative scoliosis [39].

Adult spinal scoliosis is a progressive disease that can exist for several years before it has a significant adverse impact on the patient's quality of life. For first-line treatment, many surgeons attempt non-operative measures, including nonsteroidal anti-inflammatory medications, muscle relaxants, and physical therapy, to prevent spinal deformity surgery. Injection-based therapies, including nerve root blocks, can help in pain relief, with moderate evidence in the treatment of lumbar degenerative disease [40]. Once the pain is chronic, many patients require more medications, including narcotic analgesics and neuroleptic medications. Although braces can provide temporary pain relief, they cause deconditioning of the paraspinal muscles, thus exacerbating the deformity and symptoms $[41,42]$. Conservative treatment cannot solve spinal deformity; therefore, it does not resolve all symptoms or improve the overall quality of life [39]. Surgical treatment is considered for patients who are refractory to medications as well as those who have coronal imbalances, sagittal imbalances, or neurological deterioration. However, the decision regarding surgical intervention is challenging and depends on the individual patient [43].

\section{Decision-Making for Managing Adult Spinal Deformity}

There is no definite algorithmic management for ASD. The surgical management of spinal deformity necessitates meticulous planning and careful patient selection (Fig. 2). ASD surgery is commonly associated with prolonged recovery times, high complication rates, and significant expense.

If the surgeon decides to perform surgical intervention for ASD, preoperative planning with a structured and systemic approach is important [44]. First, patientspecific radiological alignment based on the SRS-Schwab classification should be identified in order to set targets for preventing undercorrection or overcorrection [45-47]. Several factors are crucial for structural decision-making, as follows: identification of deformity drivers, setting of alignment target, and determination of flexibility. Generally, the main deformity driver is loss of LL $[10,44,48]$. These factors can be quantified by PI/LL mismatch. In most cases, this deformity starts with loss of lordosis as a driver and works on compensatory mechanisms. Therefore, PI/LL mismatch, global alignment, and PT must be evaluated during preoperative assessment. The Lafage formula is an appropriate method for achieving alignment targets. The formula incorporates PT and compensatory mechanisms and has the best correlation with optimal SVA $[15,49]$. Lateral supine radiographs are essential for assessing the flexibility of sagittal deformities. Bridwell [50] described the following flexibility categories: (1) a totally flexible deformity that corrects in the supine position, (2) a deformity that partially corrects, and (3) a totally inflexible deformity [48]. More inflexible deformities require more aggressive techniques for appropriate correction [10].

Patients must be involved in the decision-making process regarding the choice between medical or conservative intervention and operative treatment for ASD [4]. Generally, medical and conservative treatment of ASD is sup- 

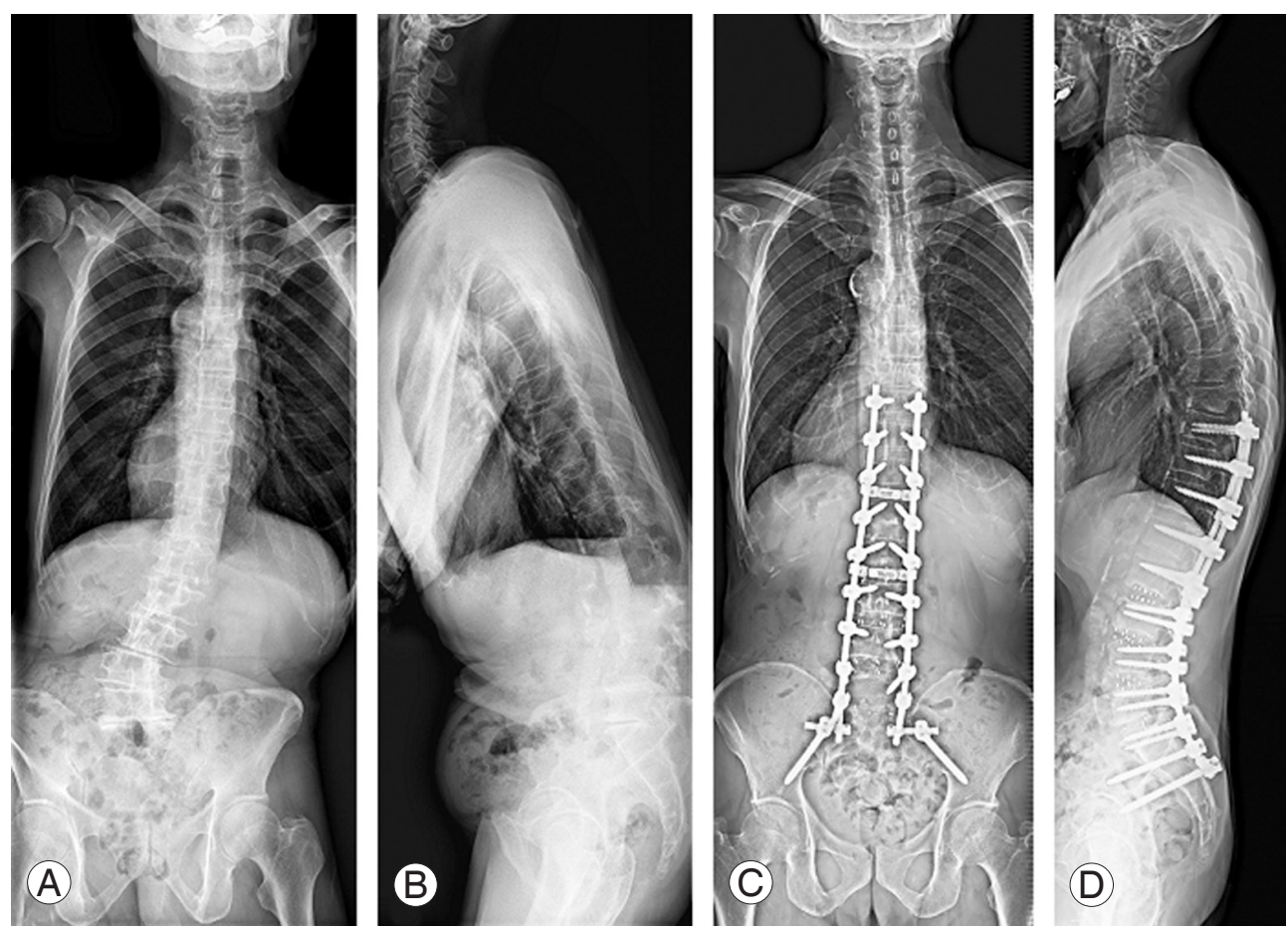

Fig. 2. Radiographs of a patient with adult spinal deformity. (A, B) Preoperative radiographs of a 73-year-old man with lumbar scoliosis of $27^{\circ}$ and positive SVA of $204 \mathrm{~mm}$. (C, D) The patient underwent deformity correction surgery using oblique lumbar interbody fusion at L2-3, L3-4, L4-5 and posterior fusion from T10 to sacrum. The lumbar scoliosis was improved to $4^{\circ}$ and SVA was improved to $24 \mathrm{~mm}$ after surgery. SVA, sagittal vertical axis.

ported by a low level of evidence. However, ASD patients are encouraged to try medical or conservative treatment before surgical correction because non-operative interventions improve mild disability and pain $[1,2,4]$. Liu et al. [51] reported that the key factor in the decision to pursue operative treatment is not the curve type or magnitude but the severity of baseline disability. Moreover, Bess et al. $[14,16]$ suggested that pain and disability are important factors that influence the decision regarding surgical management in older patients, while increased coronal plane deformity has an effect on the decision of surgical management for younger patients.

When considering surgical correction, Scheer et al. [52] found that undercorrection of sagittal deformities was correlated with worse HRQoL, while overcorrection increased the risk of proximal junctional kyphosis and other complications. As per previous research, the best practice involved overcorrection to mitigate the loss of correction that accompanies aging because alignment deterioration is more common in older patients [53]. However, this practice may not as safe because less strict alignment goals have been associated with satisfactory HRQoL levels at the population level [54].
Correction as per age-specific alignment targets is important for achieving better outcomes and preventing complications. In order to pursue appropriate correction, various techniques have been described in the literature [1]. Silva and Lenke [55] suggested the following general six-tiered hierarchy for surgical treatment: decompression alone, decompression and posterior spinal fusion with limited instrumentation, decompression with posterior spinal fusion and instrumentation of the lumbar curve, decompression with anterior and posterior instrumented spinal fusion, thoracic instrumentation and extension of the fusion, and incorporation of osteotomies specific to the curvature present. Moreover, Schwab et al. [56] proposed a simplified, anatomically based, graduated osteotomy classification system with six grades that corresponded to increasing potential for destabilization as a surgical correction method (Fig. 3).

\section{Surgical Correction Methods}

\section{Limited decompression}

For a subset of patients who present with predominantly 

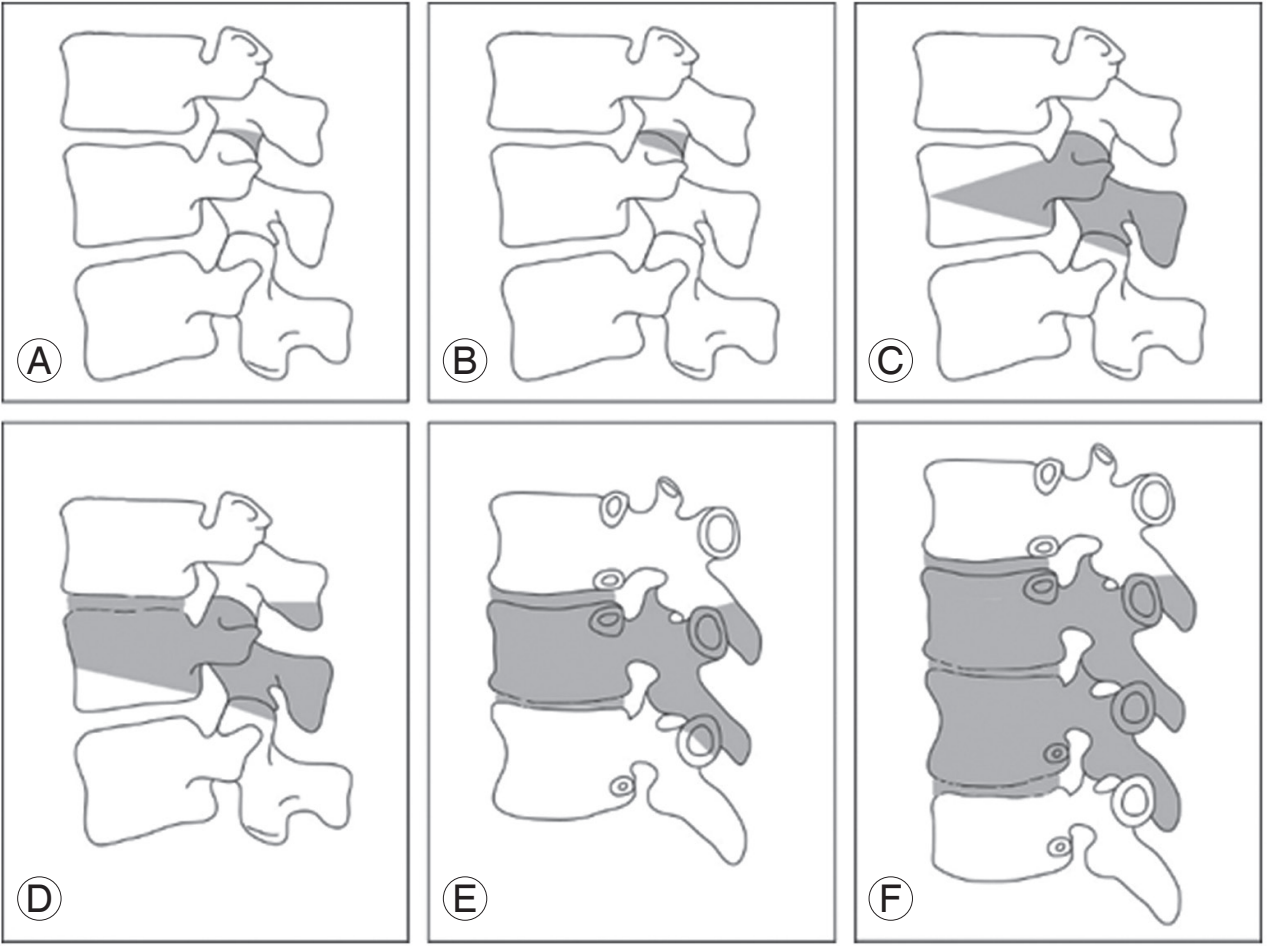

Fig. 3. Anatomically based comprehensive classification of spinal osteotomies. There are six grades, each corresponding to increasing potential for destabilization. Each grade corresponds to a resection of the following anatomic areas (shaded gray): (A) grade 1: partial facet joint; (B) grade 2: complete facet joint; (C) grade 3: pedicle and partial body; (D) grade 4: pedicle, partial body, and disc; (E) grade 5: complete vertebra and discs; and (F) grade 6: multiple vertebrae and adjacent discs.

radicular symptoms due to isolated foraminal stenosis, minimally invasive surgery (MIS) approaches have offered potential options for reducing morbidity in patients with smaller spinal deformities. MIS offers less soft-tissue dissection and easier recovery as compared to open posterior approaches [57].

Aging patients with degenerative scoliosis may benefit from a minimally invasive laminectomy with or without a foraminotomy. This procedure may provide symptomatic relief of radicular symptoms while preventing the risks associated with a large open fusion. These patients must be selected carefully because back pain and sagittal imbalance remain largely unchanged [57]. A potential side effect of this procedure is recurrent radiculopathy and mechanical instability that necessitates fusion with posterior multilevel pedicle screw placement.

\section{Limited stabilization}

Limited fusion is a common treatment of choice for single- or two-level degenerative disease. In such cases, patients have stenosis with spondylolisthesis or degen- erative disc disease. For patients with mild or moderate deformity, a short fusion construct may help in restoring alignment. For example, a patient with a lumbar flat back with an L4-L5 spondylolisthesis may be a suitable candidate for an anterior lumbar interbody fusion of L4-L5 and L5-S1 followed by posterior fusion L4-S1. In such a case, the degenerative pathology is addressed while the lordosis from L4-S1 is restored. Focal fusions can also be managed effectively with using invasive techniques.

Lateral interbody fusion (LIF) is a transpsoas approach that allows access to the anterior column of the spine without disrupting the paraspinal musculature, lowering morbidity and postoperative pain. This approach avoids the posterior tension band and is hypothesized to protect against adjacent segment disease [58]. It allows better access to the disc space as compared to posterior approaches, enabling large cage placement and improved fusion rates. With a resulting increase in the disc height, indirect neural decompression occurs. It also negates a traditional anterior approach, lowering the risk to visceral organs. Consequently, the approach can injure nerves in the lumbar plexus from the approach. Recently, the oblique 
lumbar interbody fusion (OLIF) technique has been developed and used to compensate for the shortcomings of the LIF technique. In the case of OLIF surgery, direct correction can be performed on the anterior column of the spine while preserving the psoas muscle, thus protecting the anterior and posterior muscles of the vertebral body [59].

As per a meta-analysis by Dangelmajer et al. [57] in 2014, there was no significant difference in the complication rates of MIS and open approaches. They reported a significant difference in the age of patients who underwent MIS and those who underwent open surgeries, with older patients being more suited to MIS owing to poor bone quality and comorbidities. With aging patients who have degenerative scoliosis, MIS can correct sagittal and predominantly coronal deformities, with the LIF showing the greatest change [60-62].

\section{Deformity correction}

Deformity correction is performed almost exclusively via the large posterior approach. With access to the vertebrae of interest, whose curves often include a large portion of the lumbar and thoracic spine, a large fusion construct can be built with appropriate osteotomies to enable appropriate manipulation.

Depending on the type, an osteotomy provides a certain degree of curvature correction to help in balancing the deformed spine [56]. The first two types of osteotomies involve the facet joint. There are six grades (1-6) that correlate with the degree of bone resection. The first is a partial facet excision, with removal of the inferior facet (grade 1), and the second type involves both, inferior and superior facet excision, with the removal of the ligamentum flavum and possibly other posterior elements (grade 2). Both the types of facet osteotomies need a mobile anterior column and provide limited deformity correction. A pedicle subtraction (grade 3), also called a closing wedge, is a resection of a wedge portion of the posterior vertebral body, including the pedicles, creating a hinge at the anterior column with disc sparing [56]. For a larger resection, grade 4 involves a wedge at the vertebral body, through which the endplate and a portion of one adjacent disc are removed, along with posterior elements with the pedicles. Using this type of resection, an anterior cage may be placed, particularly in cases with marked shortening.

Under certain circumstances, severely inflexible defor- mities can be managed using vertebral column resection (VCR). VCR (osteotomy grade 5 and 6) is a powerful operative method, defined as an osteotomy involving the vertebral body, intervening discs, pedicles, and all dorsal elements to create a segmental defect that requires provisional instrumentation $[63,64]$. Once the dorsal instrumentation is placed, the spine may be manipulated to achieve balance in both, the sagittal and coronal planes, shortening the length of the spinal column if necessary [65]. An interbody graft may be used to augment the anterior column and prevent unwanted spine shortening. This technically difficult procedure is associated with high complication rates, extensive operative times, and significant blood loss [66].

In order to overcome these problems, posterior multicrack osteotomy techniques have been recently developed and reported. This technique has the following merits. First, the anterior vertebral body and longitudinal ligament complex are preserved that can act as the supportive axis during correction; this prevents excessive motion around the osteotomy site. Second, multilevel osteotomies can be performed. There is no intraoperative loss or gain in the vertebral length; this can lead to spinal cord injury. Third, multilevel osteotomies can be performed with relatively minimal blood loss. Yang et al. [67] reported that spinal deformity with the fusion mass due to a previous surgery could be corrected in the coronal and sagittal planes simultaneously with posterior crack osteotomies without neurological deficits.

\section{Conclusions}

ASDs are 3D deformities; the importance of coronal and sagittal imbalance has been emphasized in recent times. Patient-specific characteristics are vital for ASD management, even if different manifestations exhibit similar pathophysiologic mechanisms, including degenerative changes and compensatory postures. Thus, ASD management should be focused on restoring age-specific harmonious alignment and should consider the comorbidities and risk factors of each patient for the prevention of catastrophic complications and improvement of HRQoL.

\section{Conflict of Interest}

No potential conflict of interest relevant to this article was reported. 


\section{References}

1. Diebo BG, Shah NV, Boachie-Adjei O, et al. Adult spinal deformity. Lancet 2019;394:160-72.

2. Youssef JA, Orndorff DO, Patty CA, et al. Current status of adult spinal deformity. Global Spine J 2013;3:51-62.

3. Schwab F, Dubey A, Gamez L, et al. Adult scoliosis: prevalence, SF-36, and nutritional parameters in an elderly volunteer population. Spine (Phila Pa 1976) 2005;30:1082-5.

4. Ames CP, Scheer JK, Lafage V, et al. Adult spinal deformity: epidemiology, health impact, evaluation, and management. Spine Deform 2016;4:310-22.

5. Good CR, Auerbach JD, O'Leary PT, Schuler TC. Adult spine deformity. Curr Rev Musculoskelet Med 2011;4:159-67.

6. Ailon T, Smith JS, Shaffrey CI, et al. Degenerative spinal deformity. Neurosurgery 2015;77 Suppl 4:S7591.

7. Kanter AS, Bradford DS, Okonkwo DO, Rengachary SS, Mummaneni PV. Thoracolumbar spinal deformity: part I. a historical passage to 1990: historical vignette. J Neurosurg Spine 2009;11:631-9.

8. Lam FC, Kanter AS, Okonkwo DO, Ogilvie JW, Mummaneni PV. Thoracolumbar spinal deformity: part II. developments from 1990 to today: historical vignette. J Neurosurg Spine 2009;11:640-50.

9. Sparrey CJ, Bailey JF, Safaee M, et al. Etiology of lumbar lordosis and its pathophysiology: a review of the evolution of lumbar lordosis, and the mechanics and biology of lumbar degeneration. Neurosurg Focus 2014;36:E1.

10. Iyer S, Sheha E, Fu MC, et al. Sagittal spinal alignment in adult spinal deformity: an overview of current concepts and a critical analysis review. JBJS Rev 2018;6:e2.

11. Bao H, Zhu F, Liu Z, et al. Coronal curvature and spinal imbalance in degenerative lumbar scoliosis: disc degeneration is associated. Spine (Phila Pa 1976) 2014;39:E1441-7.

12. Berthonnaud E, Dimnet J, Roussouly P, Labelle H. Analysis of the sagittal balance of the spine and pelvis using shape and orientation parameters. J Spinal Disord Tech 2005;18:40-7.

13. Pumberger M, Schmidt H, Putzier M. Spinal deformity surgery: a critical review of alignment and bal- ance. Asian Spine J 2018;12:775-83.

14. Bess S, Protopsaltis TS, Lafage V, et al. Clinical and radiographic evaluation of adult spinal deformity. Clin Spine Surg 2016;29:6-16.

15. Smith JS, Bess S, Shaffrey CI, et al. Dynamic changes of the pelvis and spine are key to predicting postoperative sagittal alignment after pedicle subtraction osteotomy: a critical analysis of preoperative planning techniques. Spine (Phila Pa 1976) 2012;37:84553.

16. Bess S, Schwab F, Lafage V, Shaffrey CI, Ames CP. Classifications for adult spinal deformity and use of the Scoliosis Research Society-Schwab adult spinal deformity classification. Neurosurg Clin N Am 2013;24:185-93.

17. Dagdia L, Kokabu T, Ito M. Classification of adult spinal deformity: review of current concepts and future directions. Spine Surg Relat Res 2018;3:17-26.

18. Aebi M. The adult scoliosis. Eur Spine J 2005;14:92548.

19. Naresh-Babu J, Viswanadha AK, Ito M, Park JB. What should an ideal adult spinal deformity classification system consist of?: review of the factors affecting outcomes of adult spinal deformity management. Asian Spine J 2019;13:694-703.

20. Slattery C, Verma K. Classification in brief: SRSSchwab classification of adult spinal deformity. Clin Orthop Relat Res 2018;476:1890-4.

21. Terran J, Schwab F, Shaffrey CI, et al. The SRS-Schwab adult spinal deformity classification: assessment and clinical correlations based on a prospective operative and nonoperative cohort. Neurosurgery 2013;73:55968.

22. Sebaaly A, Gehrchen M, Silvestre C, et al. Mechanical complications in adult spinal deformity and the effect of restoring the spinal shapes according to the Roussouly classification: a multicentric study. Eur Spine J 2020;29:904-13.

23. Schwab FJ, Blondel B, Bess S, et al. Radiographical spinopelvic parameters and disability in the setting of adult spinal deformity: a prospective multicenter analysis. Spine (Phila Pa 1976) 2013;38:E803-12.

24. Lee CH, Chung CK, Jang JS, Kim SM, Chin DK, Lee JK. 'Lumbar degenerative kyphosis' is not byword for degenerative sagittal imbalance: time to replace a misconception. J Korean Neurosurg Soc 2017;60:1259. 
25. Jang JS, Lee SH, Min JH, Han KM. Lumbar degenerative kyphosis: radiologic analysis and classifications. Spine (Phila Pa 1976) 2007;32:2694-9.

26. Takemitsu Y, Harada Y, Iwahara T, Miyamoto M, Miyatake Y. Lumbar degenerative kyphosis: clinical, radiological and epidemiological studies. Spine (Phila Pa 1976) 1988;13:1317-26.

27. Bridwell KH, Lenke LG, Lewis SJ. Treatment of spinal stenosis and fixed sagittal imbalance. Clin Orthop Relat Res 2001;(384):35-44.

28. Bayerl SH, Pohlmann F, Finger T, et al. The sagittal balance does not influence the 1 year clinical outcome of patients with lumbar spinal stenosis without obvious instability after microsurgical decompression. Spine (Phila Pa 1976) 2015;40:1014-21.

29. Lee CS, Kim YT, Kim E. Clinical study of lumbar degenerative kyphosis. J Korean Soc Spine Surg 1997;4:27-35.

30. Shiba Y, Taneichi H, Inami S, Moridaira H, Takeuchi D, Nohara Y. Dynamic global sagittal alignment evaluated by three-dimensional gait analysis in patients with degenerative lumbar kyphoscoliosis. Eur Spine J 2016;25:2572-9.

31. Bae J, Theologis AA, Jang JS, Lee SH, Deviren V. Impact of fatigue on maintenance of upright posture: dynamic assessment of sagittal spinal deformity parameters after walking 10 minutes. Spine (Phila $\mathrm{Pa}$ 1976) 2017;42:733-9.

32. Taneichi H. Update on pathology and surgical treatment for adult spinal deformity. J Orthop Sci 2016;21:116-23.

33. Doherty JH. Complications of fusion in lumbar scoliosis: proceedings of the Scoliosis Research Society. J Bone Joint Surg Am 1973;55:438.

34. Potter BK, Lenke LG, Kuklo TR. Prevention and management of iatrogenic flatback deformity. J Bone Joint Surg Am 2004;86:1793-808.

35. Boody BS, Rosenthal BD, Jenkins TJ, Patel AA, Savage JW, Hsu WK. Iatrogenic flatback and flatback syndrome: evaluation, management, and prevention. Clin Spine Surg 2017;30:142-9.

36. Booth KC, Bridwell KH, Lenke LG, Baldus CR, Blanke KM. Complications and predictive factors for the successful treatment of flatback deformity (fixed sagittal imbalance). Spine (Phila Pa 1976) 1999;24:171220.

37. Faraj SS, Holewijn RM, van Hooff ML, de Kleuver M,
Pellise F, Haanstra TM. De novo degenerative lumbar scoliosis: a systematic review of prognostic factors for curve progression. Eur Spine J 2016;25:2347-58.

38. Wong E, Altaf F, Oh LJ, Gray RJ. Adult degenerative lumbar scoliosis. Orthopedics 2017;40:e930-9.

39. Graham RB, Sugrue PA, Koski TR. Adult degenerative scoliosis. Clin Spine Surg 2016;29:95-107.

40. Watters WC 3rd, Resnick DK, Eck JC, et al. Guideline update for the performance of fusion procedures for degenerative disease of the lumbar spine: part 13: injection therapies, low-back pain, and lumbar fusion. J Neurosurg Spine 2014;21:79-90.

41. Van Dam BE. Nonoperative treatment of adult scoliosis. Orthop Clin North Am 1988;19:347-51.

42. Smith JS, Shaffrey CI, Berven S, et al. Improvement of back pain with operative and nonoperative treatment in adults with scoliosis. Neurosurgery 2009;65:86-94.

43. Glassman SD, Schwab FJ, Bridwell KH, Ondra SL, Berven S, Lenke LG. The selection of operative versus nonoperative treatment in patients with adult scoliosis. Spine (Phila Pa 1976) 2007;32:93-7.

44. Kim YJ, Hyun SJ, Cheh G, Cho SK, Rhim SC. Decision making algorithm for adult spinal deformity surgery. J Korean Neurosurg Soc 2016;59:327-33.

45. Moal B, Schwab F, Ames CP, et al. Radiographic outcomes of adult spinal deformity correction: a critical analysis of variability and failures across deformity patterns. Spine Deform 2014;2:219-25.

46. Schwab FJ, Patel A, Shaffrey CI, et al. Sagittal realignment failures following pedicle subtraction osteotomy surgery: are we doing enough?: clinical article. J Neurosurg Spine 2012;16:539-46.

47. Lafage V, Ames C, Schwab F, et al. Changes in thoracic kyphosis negatively impact sagittal alignment after lumbar pedicle subtraction osteotomy: a comprehensive radiographic analysis. Spine (Phila $\mathrm{Pa}$ 1976) 2012;37:E180-7.

48. Smith JS, Shaffrey CI, Ames CP, Lenke LG. Treatment of adult thoracolumbar spinal deformity: past, present, and future. J Neurosurg Spine 2019;30:55167.

49. Passias PG, Bortz CA, Segreto FA, et al. Pelvic incidence affects age-adjusted alignment outcomes in a population of adult spinal deformity. Clin Spine Surg 2020 Jun 19 [Epub]. https://doi.org/10.1097/ BSD.0000000000001025.

50. Bridwell KH. Decision making regarding Smith-Pe- 
tersen vs. pedicle subtraction osteotomy vs. vertebral column resection for spinal deformity. Spine (Phila Pa 1976) 2006;31(19 Suppl):S171-8.

51. Liu S, Schwab F, Smith JS, et al. Likelihood of reaching minimal clinically important difference in adult spinal deformity: a comparison of operative and nonoperative treatment. Ochsner J 2014;14:67-77.

52. Scheer JK, Lafage R, Schwab FJ, et al. Under correction of sagittal deformities based on age-adjusted alignment thresholds leads to worse health-related quality of life whereas over correction provides no additional benefit. Spine (Phila Pa 1976) 2018;43:38893.

53. Cho KJ, Kim KT, Kim WJ, et al. Pedicle subtraction osteotomy in elderly patients with degenerative sagittal imbalance. Spine (Phila Pa 1976) 2013;38:E15616.

54. Lafage R, Schwab F, Glassman S, et al. Age-adjusted alignment goals have the potential to reduce PJK. Spine (Phila Pa 1976) 2017;42:1275-82.

55. Silva FE, Lenke LG. Adult degenerative scoliosis: evaluation and management. Neurosurg Focus 2010;28:E1.

56. Schwab F, Blondel B, Chay E, et al. The comprehensive anatomical spinal osteotomy classification. Neurosurgery 2014;74:112-20.

57. Dangelmajer S, Zadnik PL, Rodriguez ST, Gokaslan ZL, Sciubba DM. Minimally invasive spine surgery for adult degenerative lumbar scoliosis. Neurosurg Focus 2014;36:E7.

58. Cheh G, Bridwell KH, Lenke LG, et al. Adjacent segment disease followinglumbar/thoracolumbar fusion with pedicle screw instrumentation: a minimum 5-year follow-up. Spine (Phila Pa 1976) 2007;32:22537.

59. Verma R, Virk S, Qureshi S. Interbody fusions in the lumbar spine: a review. HSS J 2020;16:162-7.
60. Iwamae M, Matsumura A, Namikawa T, et al. Surgical outcomes of multilevel posterior lumbar interbody fusion versus lateral lumbar interbody fusion for the correction of adult spinal deformity: a comparative clinical study. Asian Spine J 2020;14:421-9.

61. Kim WJ, Lee JW, Park KY, Chang SH, Song DG, Choy WS. Treatment of adult spinal deformity with sagittal imbalance using oblique lumbar interbody fusion: can we predict how much lordosis correction is possible? Asian Spine J 2019;13:1017-27.

62. Kim WJ, Lee JW, Kim SM, et al. Precautions for combined anterior and posterior long-level fusion for adult spinal deformity: perioperative surgical complications related to the anterior procedure (oblique lumbar interbody fusion). Asian Spine J 2019;13:82331.

63. Helgeson MD, Bevevino AJ, Hilibrand AS. Update on the evidence for adjacent segment degeneration and disease. Spine J 2013;13:342-51.

64. Yang C, Zheng Z, Liu H, Wang J, Kim YJ, Cho S. Posterior vertebral column resection in spinal deformity: a systematic review. Eur Spine J 2016;25:2368-75.

65. Schwab F, Lafage V, Farcy JP, et al. Surgical rates and operative outcome analysis in thoracolumbar and lumbar major adult scoliosis: application of the new adult deformity classification. Spine (Phila Pa 1976) 2007;32:2723-30.

66. Lenke LG, Newton PO, Sucato DJ, et al. Complications after 147 consecutive vertebral column resections for severe pediatric spinal deformity: a multicenter analysis. Spine (Phila Pa 1976) 2013;38:11932.

67. Yang JH, Suh SW, Cho WT, Hwang JH, Hong JY, Modi HN. Effect of posterior multilevel vertebral osteotomies on coronal and sagittal balance in fused scoliosis deformity caused by previous surgery: preliminary results. Spine (Phila Pa 1976) 2014;39:18409. 